03. 1;10

\title{
Зависимость звукового удара от взаимного расположения тел в сверхзвуковом потоке
}

\author{
(C) А.В. Потапкин, Д.Ю. Москвичев \\ Институт теоретической и прикладной механики им. С.А. Христиановича СО РАН, \\ Новосибирск, Россия \\ E-mail: potapkin@itam.nsc.ru
}

Поступило в Редакцию 11 октября 2019 г.

В окончательной редакции 11 октября 2019 г.

Принято к публикации 20 декабря 2019 г.

\begin{abstract}
Представлены результаты расчетов уровня звукового удара, возникающего при обтекании сверхзвуковым потоком воздуха двух тел: диска и тонкого тела вращения. Тела расположены друг за другом. Тонкое тело находится в аэродинамической тени от диска. Число Маха набегающего потока равно 2. Расчеты выполнены с помощью комбинированного метода „тел-фантомов“. Изменением положения диска и его размера можно добиться снижения уровня звукового удара. По результатам расчетов дано описание газодинамических факторов, влияющих на уровень звукового удара.
\end{abstract}

Ключевые слова: ударные волны, звуковой удар, тонкое тело, аэродинамическая тень, метод „тел-фантомов“.

DOI: 10.21883/PJTF.2020.06.49165.18071

В настоящее время разрабатываются различные способы снижения уровня звукового удара от сверхзвукового самолета. Обзоры исследований по проблеме звукового удара можно найти в работах [1,2]. Разработка способов предполагает знание газодинамических факторов, обеспечивающих снижение уровня звукового удара от тела в сверхзвуковом потоке. Одним из активных способов воздействия на звуковой удар является способ изменения параметров набегающего потока с помощью аэродинамических поверхностей перед телом $[3,4]$. В настоящей работе рассматриваются газодинамические факторы, обеспечивающие изменение уровня звукового удара от тонкого тела, находящегося в аэродинамической тени другого тела. Поверхность тонкого тела вращения в размерных величинах задавалась формулой $r=0.2\left(x-x^{2} / L\right), 0 \leq x \leq L$, где $L-$ длина тела, $x$ - продольная координата, отсчитываемая от носика тела вниз по потоку, $r$ - радиальная координата поверхности тела. Аэродинамическая тень создавалась диском, расположенным перед тонким телом поперек сверхзвукового набегающего потока.

В работе использовались следующие обозначения: $p_{0}$ - статическое давление в набегающем потоке, $\Delta p-$ избыточное давление, $\mathrm{M}_{0}$ - число Маха набегающего потока воздуха, $d$ - диаметр диска. Расчеты поля течения выполнялись в декартовой или цилиндрической системе координат. При расчетах трехмерного течения ближнего поля использовалась декартова система координат $\{x, y, z\}: x-$ продольная координата, отсчитываемая от носика тела вниз по потоку, $y-$ вертикальная координата, $\{x y\}-$ плоскость симметрии, $z$ - боковое удаление от плоскости симметрии. При выполнении расчетов ближнего поля течения, имеюще- го осевую симметрию, использовалась цилиндрическая система координат $\{r, x\}: r$ - радиальная координата, $x$ - продольная координата, отсчитываемая от носика тела вниз по потоку.

Расчеты ближнего поля течения проводились с помощью пакета программ ANSYS Fluent с использованием модели вязкого теплопроводного газа. Параметры расчета: $\mathrm{M}_{0}=2, L=40 \mathrm{~m}, p_{0}=5474.87 \mathrm{~Pa}$, $T_{0}=216.65 \mathrm{~K}$, где $T_{0}$ - статическая температура воздуха в набегающем потоке.

Параметры ближнего поля использовались в качестве начальных данных для расчетов дальнего поля. Расчеты дальнего поля выполнялись по методу „тел-фантомов“ [5] с использованием аналитических решений Ландау [6], Уизема [7] и Рао [8] в системе координат с осевой симметрией. В расчетах трехмерного течения начальные данные выбирались на азимутальной плоскости $\{\varphi=$ const $\}$ в цилиндрической системе координат $\{r, \varphi, x\}$. В этом случае в каждой плоскости $\{\varphi=$ const $\}$ строилось соответствующее „тело-фантом“ и дальнее поле течения рассчитывалось независимо в каждой азимутальной плоскости в цилиндрической системе координат $\{r, x\}$ с осевой симметрией. Осью симметрии была ось $x$. Пространственная картина течения получалась при выполнении расчетов в нескольких азимутальных плоскостях. На возможность такого подхода указывалось в монографии Уизема [9], и это проверялось в работе [5] при решении пространственных задач.

Тестирование метода „тел-фантомов“ для заостренных и затупленных тел выполнено в работах $[4,5]$. На рис. $1, a$ представлено сравнение результатов расчетов с результатами экспериментов в баллистической трассе [10]. Использованы следующие обозначения: $D$ - диаметр 


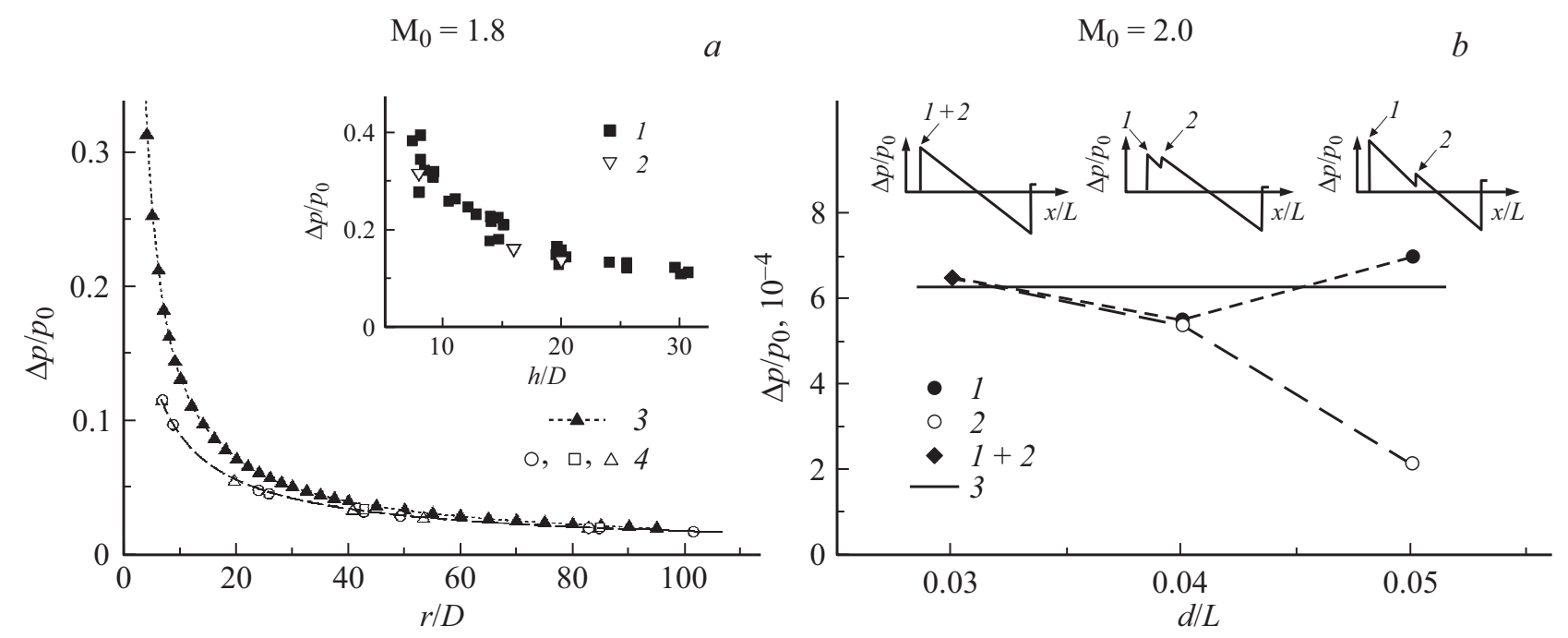

Рис. 1. $a$ - тестирование метода „тел-фантомов“. 1 - измерения давления на мерной пластине за отраженной головной ударной волной в баллистической трассе [10], 2 - 3D-расчеты с помощью ANSYS Fluent, 3 - 2D-расчеты интенсивности головной ударной волны с помощью ANSYS Fluent, 4 - расчеты по методу „тел-фантомов“ [4]. $b$ - расчеты дальнего поля для тонкого тела с диском. Относительная интенсивность ударных волн и профили относительного избыточного давления: 1 - ударная волна от диска, 2 - ударная волна от тела (висячий скачок), $1+2-$ ударная волна, образовавшаяся после слияния ударных волн от диска и от тела, 3 - относительная интенсивность головной ударной волны от тонкого тела без диска.

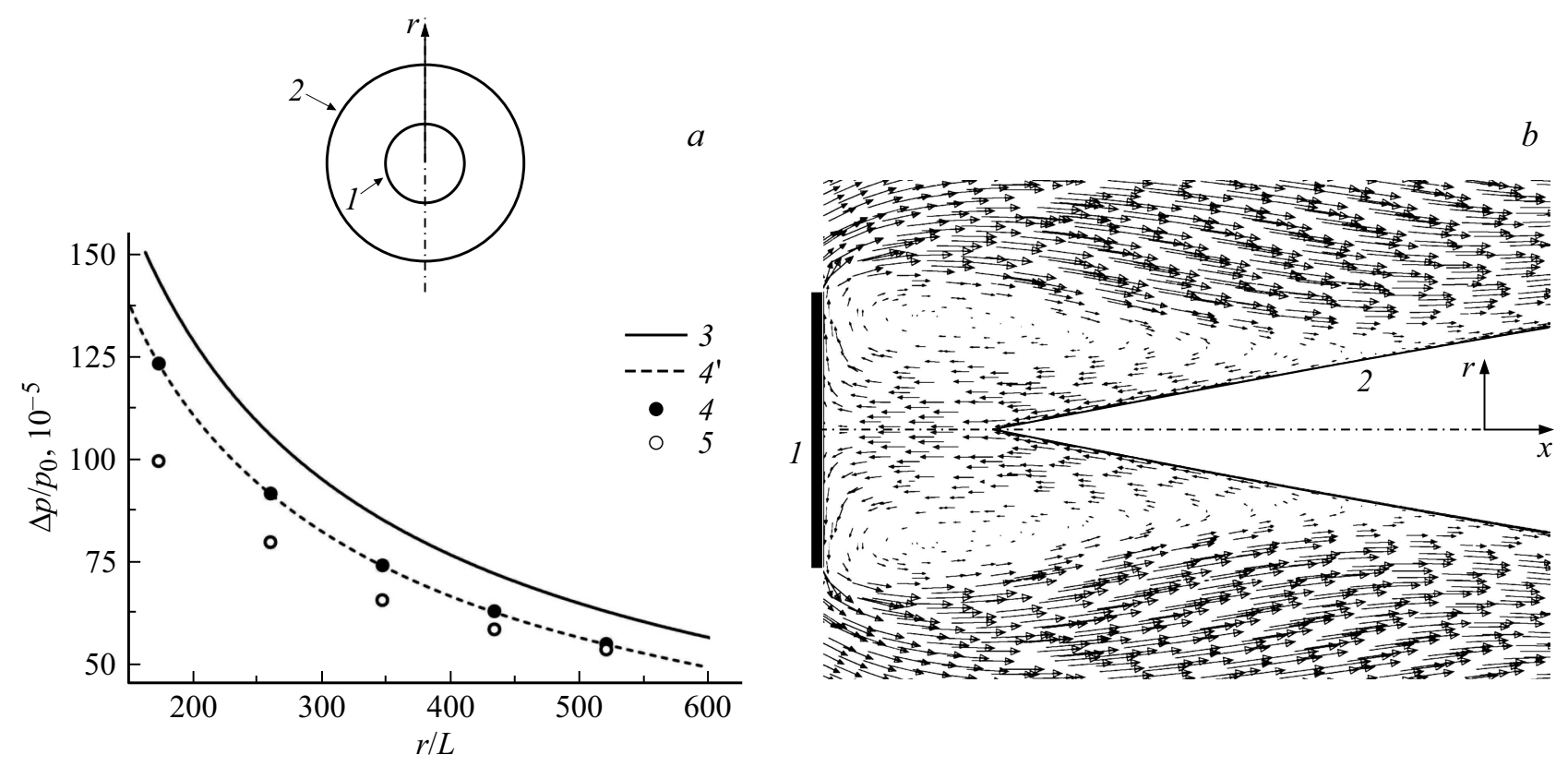

Рис. 2. Тело с диском с $d / L=0.04$ (осевая симметрия). Относительная интенсивность ударных волн $(a)$ и векторное поле скорости потока $(b) .1$ - диск, 2 - тонкое тело вращения, 3 - относительная интенсивность головной ударной волны от тонкого тела без диска, $4^{\prime}$ - относительная интенсивность ударной волны от диска без тела, 4 - относительная интенсивность ударной волны от диска, 5 - относительная интенсивность висячего скачка.

шара, $r$ - радиальное расстояние от траектории полета шара, $h$ - расстояние от траектории полета до мерной пластины. Тестовые расчеты показали, что метод „тел-фантомов“ применим для вычисления параметров ударных волн в дальнем поле как для заостренных, так и для затупленных тел. На рис. $1, b$ приведены расчеты параметров дальнего поля течения для тела с диском.
Расстояние между диском и телом задавалось равным 0.025L. Перед диском возникает отошедшая ударная волна 1. За диском формируется аэродинамическая тень с дозвуковым возвратным течением. Во внешнем потоке над областью аэродинамической тени возникает ударная волна от тела 2, которая формируется как висячий скачок. Результаты расчетов относительной интенсивности 


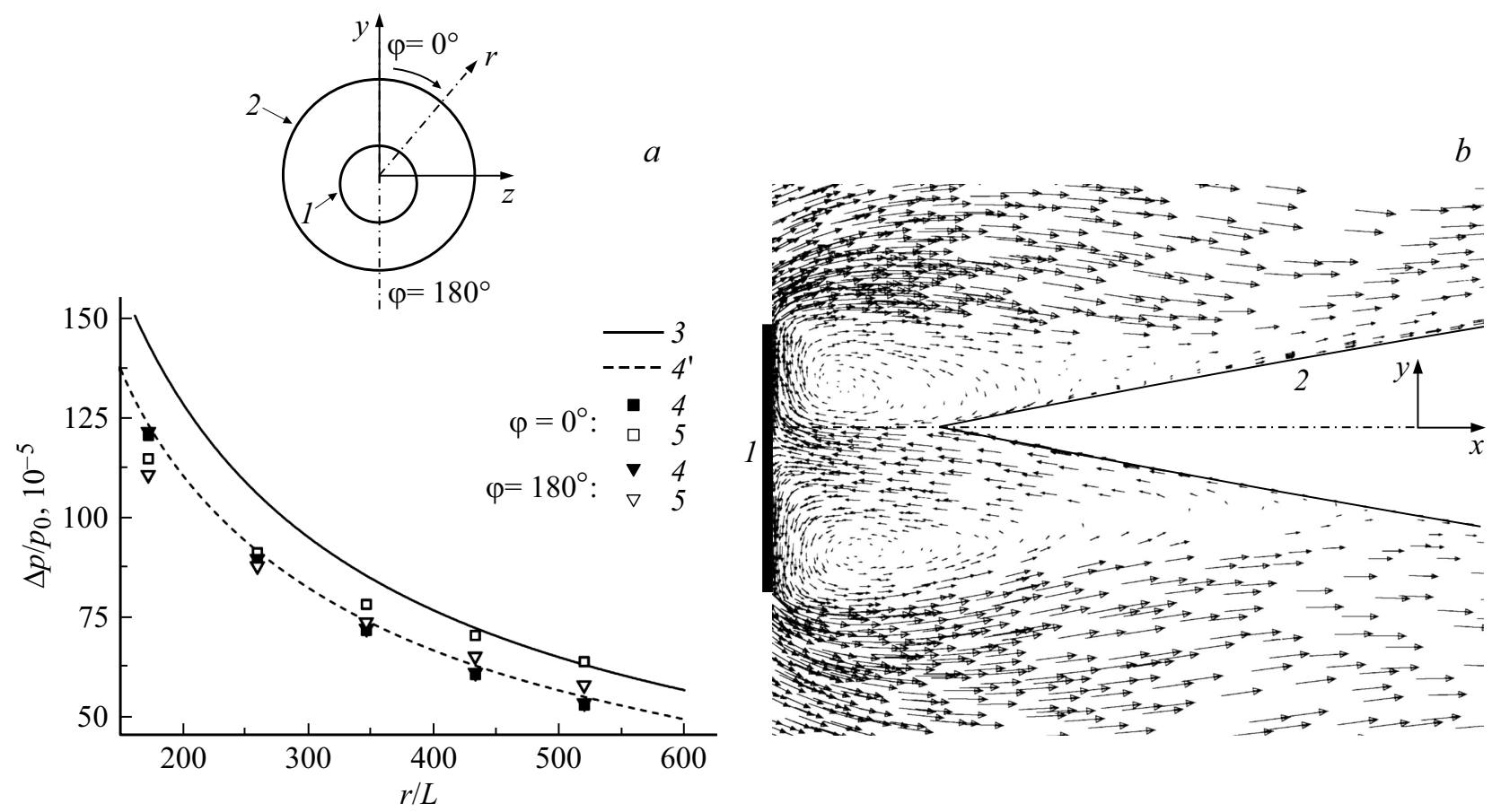

Рис. 3. Тело с диском с $d / L=0.04$ (течение без осевой симметрии). Относительная интенсивность ударных волн ( $a$ ) и векторное поле скорости потока $(b) .1$ - диск, 2 - тонкое тело вращения, 3 - относительная интенсивность головной ударной волны от тонкого тела без диска, $4^{\prime}$ - относительная интенсивность ударной волны от диска без тела, 4 - относительная интенсивность ударной волны от диска для разных азимутальных плоскостей, 5 - относительная интенсивность висячего скачка для разных азимутальных плоскостей.

ударных волн для тела с дисками разного диаметра и соответствующие профили относительного избыточного давления за ударными волнами показаны для расстояния $r / L \sim 520$. Для сравнения на рисунке представлена относительная интенсивность головной ударной волны от тонкого тела без диска. Ударная волна, которая образуется после слияния ударных волн, обозначена $1+2$. Видно, что размер диска и соответственно размер аэродинамической тени влияют на уровень звукового удара в дальнем поле. Для диска с диаметром $d=0.03 L$ ударная волна от тела догоняет ударную волну от диска, сливается с ней и в дальнем поле звуковой удар становится сильнее, чем от исходного тонкого тела. В ближнем поле интенсивности ударных волн от диска и от тела были меньше интенсивности головной ударной волны от тонкого тела без диска, что позволяло делать предварительный прогноз о снижении уровня звукового удара в дальнем поле. Использование данных ближнего поля для прогноза уровня звукового удара в дальнем поле без точного расчета чаще всего оказывается необоснованным и дает ошибочные результаты. Диск с диаметром $d=0.04 L$ создает такую аэродинамическую тень, что ударные волны от диска и от тела не сливаются на расстоянии $r / L \sim 520$, и получается уменьшение звукового удара. При дальнейшем увеличении диаметра диска $(d=0.05 L)$ аэродинамическая тень увеличивается и возрастает интенсивность ударной волны от диска. Интенсивность ударной волны от тела уменьшается, но уровень звукового удара уже определяется ударной волной от диска и превосходит уровень звукового удара от исходного тела.

На рис. 2 представлены результаты расчетов для тела с диском при $d=0.04 L$. На рис. 2, $a$ показано фронтальное изображение тела с диском и результаты расчетов относительной интенсивности ударных волн на различных расстояниях $r / L$ от оси симметрии $x$. На рис. $2, b$ в плоскости $\{r x\}$ показано векторное поле скорости потока. За диском возникает аэродинамическая тень с дозвуковым возвратным течением. Относительная интенсивность висячего скачка над этой областью на всех расстояниях $r / L$ меньше относительной интенсивности головной ударной волны от тела без диска. Относительная интенсивность ударной волны от диска совпадает с интенсивностью ударной волны от диска без тела. Как показали эти расчеты, в дальнем поле уменьшение уровня звукового удара может достигать величины $12 \%$ и более. На расстояниях $r / L>520$ висячий скачок будет догонять ударную волну от диска и произойдет их слияние.

На рис. 3 приведены результаты расчетов для тела с диском с $d=0.04 L$, смещенным в отрицательном направлении вдоль оси $y$ на расстояние $0.005 L$. На рис. $3, a$ представлено фронтальное изображение тела со смещенным диском и результаты расчетов относительной интенсивности ударных волн на различных расстояниях $r / L$ от оси $x$ в азимутальных плоскостях $\left\{\varphi=0^{\circ}\right\}$ и 
$\left\{\varphi=180^{\circ}\right\}$. На рис. $3, b$ показано векторное поле скорости потока в плоскости $\{x y\}$. В результате смещения диска размер аэродинамической тени в верхней части пространства на поверхности тела стал меньше, а в нижней части больше по сравнению с осесимметричным случаем. Можно было ожидать изменения уровня звукового удара в соответствии с изменением размера аэродинамической тени, т.е. уменьшения уровня удара в нижней части пространства и увеличения уровня удара в верхней части пространства. Но расчеты поля течения вблизи тела показали, что при изменении компоновки возникают азимутальные течения, вследствие чего давление на верхней и нижней поверхностях тела становится выше давления, полученного для осесимметричного случая. Изменение компоновки привело к усилению уровня удара, поскольку интенсивность висячего скачка, следующего за ударной волной от диска, возросла по сравнению с осесимметричным случаем.

В результате расчетов установлено, что существуют такие размеры и положения препятствий перед телом, которые обеспечивают уменьшение звукового удара на больших расстояниях от тела за счет создания аэродинамической тени.

Результаты работы показывают, что перемещение элементов компоновки с плохообтекаемыми телами, создающими ударные волны, не гарантирует снижения уровня звукового удара. Такое перемещение не обеспечивает автоматическое уменьшение звукового удара в выбранной азимутальной плоскости. Наоборот, можно получить обратный эффект и усилить звуковой удар, что является следствием развития азимутальных течений.

Два газодинамических фактора определяют уровень удара от двух тел в сверхзвуковом потоке: 1) аэродинамическая тень за первым телом, в которой находится второе тело; 2) азимутальные течения, которые возникают вблизи поверхности тела при асимметрии течения.

\section{Финансирование работы}

Работа выполнена в рамках Программы фундаментальных научных исследований государственных академий наук на 2013-2020 гг. (проект AAAA-A17-117030610126-4).

\section{Конфликт интересов}

Авторы заявляют, что у них нет конфликта интересов.

\section{Список литературы}

[1] Benson L.R. Quieting the boom: the shaped sonic boom demonstrator and the quest for quiet supersonic flight. NASA, 2013. $401 \mathrm{p}$.

[2] Sun Y., Smith H. // Prog. Aerosp. Sci. 2017. V. 90. P. 12-38. https://doi.org/10.1016/j.paerosci.2016.12.003
[3] Potapkin A.V., Moskvichev D.Yu. Ability to control the sonic boom level by means of aerodynamic surfaces // AIP Conf. Proc. 2017. V. 1893. P. 030156. https://doi.org/10.1063/1.5007614

[4] Potapkin A.V., Moskvichev D.Yu. // Shock Waves. 2018. V. 28. N 6. P. 1239-1249. https://doi.org/10.1007/s00193-018-0817-1

[5] Потапкин А.В., Москвичев Д.Ю. // ПМТФ. 2011. Т. 52. № 2. C. $15-26$

[6] Ландау Л.Д. // ПММ. 1945. Т. 9. В. 4. С. 286-292.

[7] Whitham G.B. // Commun. Pure Appl. Math. 1952. V. 5. N 3. P. 301-348. https://doi.org/10.1002/cpa.3160050305

[8] Rao P.S. // Aeronaut. Q. 1956. V. 7. N 1. P. 21-44. https://doi.org/10.1017/S0001925900010118

[9] Уизем Джс. Линейные и нелинейные волны. М.: Мир, 1977. $662 \mathrm{c}$.

[10] Красильщиков А.П., Гурьяшкин Л.П. Экспериментальные исследования тел вращения в гиперзвуковых потоках. М.: Физматлит, 2007. 208 с. 